\title{
Effect of Statin Treatment in Patients With Acute Myocardial Infarction and Left Ventricular Systolic Dysfunction According to the Level of High-Sensitivity C-Reactive Protein
}

\author{
Hae Chang JeOng, ${ }^{1}$ MD, Youngkeun Ahn, ${ }^{1}$ MD, Keun-Ho PARK, ${ }^{1}$ MD, Doo Sun Sim,,${ }^{1}$ MD, \\ Young Joon Hong, ${ }^{1}$ MD, Ju Han KIM, ${ }^{1}$ MD, Myung Ho JeOnG, ${ }^{1}$ MD, Young Jo KIm, ${ }^{2}$ MD, \\ Shung Chull ChaE, ${ }^{3} \mathrm{MD}$, Myeong Chan Cho, ${ }^{4} \mathrm{MD}$, Jei Keon ChaE, ${ }^{5} \mathrm{MD}$, Chong Jin Kim, ${ }^{6} \mathrm{MD}$, \\ Seung-Woon Rha, ${ }^{7}$ MD, Yang Soo JANG, ${ }^{8}$ MD, Seok Kyu OH, ${ }^{9}$ MD, In Whan SeOnG, ${ }^{10}$ MD, \\ and Kwang Soo CHA, ${ }^{11} \mathrm{MD}$, for the Korea Acute Myocardial Infarction Registry (KAMIR) Investigators
}

\begin{abstract}
SUMMARY
The effects of statins on the prognosis of patients with left ventricular (LV) systolic dysfunction remain controversial. The aim of this study was to assess the effect of statin treatment on clinical outcomes in acute myocardial infarction (AMI) patients with LV systolic dysfunction. A total of 5,119 AMI patients with LV ejection fraction less than 50\% on the initial echocardiogram were analyzed in the Korean Acute Myocardial Infarction Registry. The study population was divided into 4 groups according to the level of high sensitivity C-reactive protein (hs-CRP) and statin treatment: low hsCRP (hs-CRP $\leq 2.0 \mathrm{mg} / \mathrm{L}$ ) and high hs-CRP (hs-CRP $>2 \mathrm{mg} / \mathrm{L}$ ) with or without statin therapy. We evaluated the incidence of major adverse cardiac events (MACEs) including cardiac death, reinfarction, target lesion revascularization, and coronary artery bypass grafting during a 12-month period in each group. Statin therapy did not significantly prevent the MACEs in the low hs-CRP groups (with statin: $10.1 \%$ versus without statin: $12.0 \%, P=0.249$ ). In the high hs-CRP groups, however, the incidence of MACEs was significantly decreased with statin treatment (with statin: $11.3 \%$, without statin: $20.8 \%, P<0.001$ ). These findings were consistently observed in all subgroups of the high-hs CRP group, including the subgroup with an LV ejection fraction less than 40\%. In a multivariable logistic regression analysis of the high hs-CRP group, lack of statin therapy was a significant predictor of MACE incidence (odds ratio: 1.573, 95\% confidence interval: $1.079-2.293, P=0.018$ ). The statin treatment was associated with better outcome in AMI and LV dysfunction patients with hs-CRP $\geq 2 \mathrm{mg} / \mathrm{dL}$. (Int Heart J 2014; 55: 106-112)
\end{abstract}

Key words: Coronary artery disease, Inflammation, Prognosis

I nflammation is thought to play an important role in the pathophysiology of acute myocardial infarction (AMI) and left ventricular (LV) dysfunction. ${ }^{1-4)}$ Ischemic injury promotes myocardial inflammation and pro-inflammatory cytokines stimulate $\mathrm{C}$-reactive protein (CRP) leading to cardiac remodeling with LV dysfunction as its clinical manifestation. $^{5,6)}$ Yet, the mechanisms of LV dysfunction post-AMI remain poorly understood. Indeed, high-sensitivity CRP (hsCRP) is a marker of inflammation activated early after AMI and is associated with LV dysfunction, and is predictive of worse clinical outcomes. ${ }^{7)}$

In addition to reducing cholesterol levels, statins also have an anti-inflammatory action. ${ }^{8,9)}$ How much of the benefit of statins, if any, is due to this anti-inflammatory action in AMI patients with decreased left ventricular ejection fraction (LVEF) is uncertain.

Conversely, a low level of cholesterol is an independent predictor of a poor prognosis in patients with LV dysfunction. $^{10,11)}$ Therefore, AMI with LV dysfunction is a state characterized by inflammation in which a low level of cholesterol is associated with worse outcome, ${ }^{12)}$ in which the usual epidemiological relationship between cholesterol and cardiovascular outcomes is reversed and thus represents an excellent disease model in which to test the statin anti-inflammatory hypothesis. ${ }^{13,14)}$ We therefore investigated whether statin therapy and hs-CRP levels were associated with the long-term clinical outcome in AMI patients with decreased LV systolic function.

From the ${ }^{1}$ Chonnam National University Hospital, Gwangju, ${ }^{2}$ Yeongnam University Hospital, ${ }^{3}$ Kyungpook National University Hospital, Daegu, ${ }^{4}$ Chungbuk National University Hospital, Cheongju, ${ }^{5}$ Chonbuk National University Hospital, Jeonju, ${ }^{6}$ Kyung Hee University Hospital, ${ }^{7}$ Korea University Guro Hospital, ${ }^{8}$ Yonsei University Severans Hospital, Seoul, ${ }^{9}$ Wonkwang University Hospital, Iksan, ${ }^{10}$ Chungnam Nat University Hospital, Daejeon, and ${ }^{11}$ Pusan National University Hospital, Busan, Korea.

This study was supported by a grant from the National Research Foundation of Korea Grant funded by the Korean Government (MEST), Republic of Korea (20100020261), and the Korean Health Technology R\&D Project, Ministry of Health \& Welfare, Republic of Korea (HI12C0199).

Address for correspondence: Youngkeun Ahn, MD, Department of Cardiology, Cardiovascular Center, Chonnam National University Hospital, 671 Jebongro, Donggu, Gwangju 501-757, Korea. E-mail: cecilyk@ hanmail.net

Received for publication August 6, 2013. Revised and accepted September 15, 2013.

Released advance online J-STAGE March 14, 2014.

All rights are reserved to the International Heart Journal Association. 


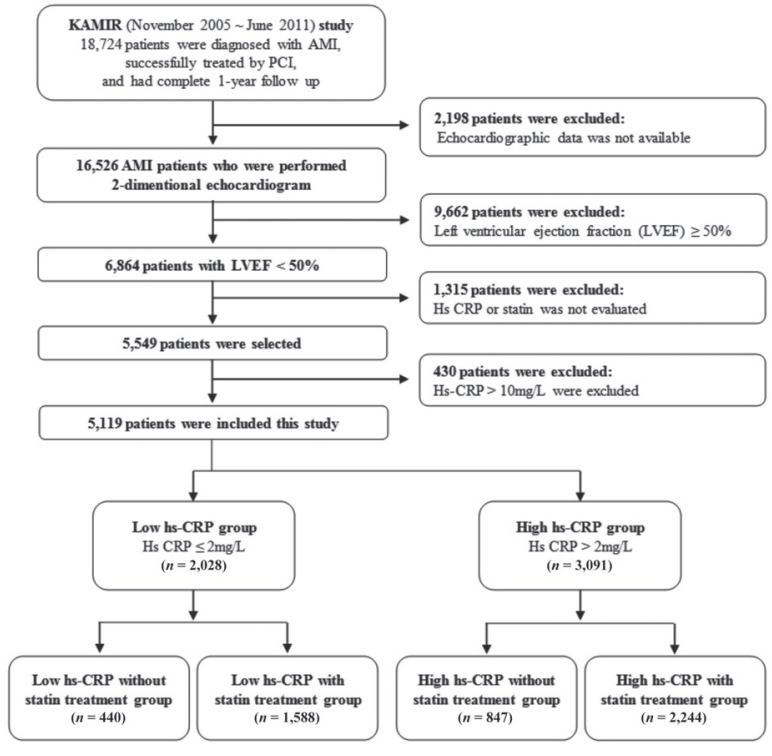

Figure 1. Study flow chart. 16,526 AMI patients who underwent 2-dimensional echocardiogram. AMI indicates acute myocardial infarction; hs-CRP, high-sensitivity C-reactive protein; KAMIR, Korea Acute Myocardial Infarction Registry; and PCI, percutaneous coronary intervention.

\section{Methods}

Korea Acute Myocardial Infarction Registry (KAMIR): The Korea AMI Registry (KAMIR) is a Korean prospective, multicenter, and observational online registry started in November 2005 that was designed to reflect "real-world" practice in Korean AMI patients with support from the Korean Circulation Society. The general characteristics of KAMIR have been described elsewhere. ${ }^{15)}$ The study protocol was approved by the ethics committee at each participating institution, and all patients were informed about their participation in the registry.

Study population: For the present study, we enrolled 18,724 patients from November 2005 to June 2011. Patients were eligible for enrollment if they presented with AMI within 12 hours after symptom onset, if they were successfully treated by percutaneous coronary intervention (PCI) with or without stent implantation, and if they survived the hospitalization period and had completed a one-year clinical follow-up period. The following patients were excluded sequentially: patients with LVEF greater than $50 \%$, patients lacking information on hsCRP levels and statin administration, and patients with hs-CRP greater than $10 \mathrm{mg} / \mathrm{L}$ and with active infections, tumors, or inflammatory disease. A total of 5,119 patients were included in the study. We divided the population using a hs-CRP value of $2.0 \mathrm{mg} / \mathrm{L}$ as the cutoff point. Thus, the low hs-CRP group consisted of patients with hs-CRP $\leq 2.0 \mathrm{mg} / \mathrm{L}(n=2,028)$ and the high hs-CRP group consisted of patients with hs-CRP $>2 \mathrm{mg} /$ $\mathrm{L}(n=3,091)$. Both hs-CRP groups were then broken into subgroups according to the statin treatment: a low hs-CRP without statin group $(n=440)$, low hs-CRP with statin group ( $n=$ $1,588)$, high hs-CRP without statin group $(n=847)$, and high hs-CRP with statin group $(n=2,244)$ (Figure 1$)$. The statins were started before or immediately after PCI and continued in the hospital period, at discharge, and throughout the follow-up period. The 29 patients in whom statin treatment was discon- tinued during the study period were excluded from the analysis. Statin treatment before the AMI did not have an effect on assignment to the groups. The choice of type and dose of statin was left to the doctor's discretion. The statins prescribed were simvastatin $(20,40,80 \mathrm{mg})$, rosuvastatin $(5,10,20 \mathrm{mg})$, pravastatin $(5,10,20,40 \mathrm{mg})$, pitavastatin (2 mg), lovastatin (20 $\mathrm{mg})$, atorvastatin $(10,20,40,80 \mathrm{mg})$, and fluvastatin $(20,40$, $80 \mathrm{mg}$ ).

Study definitions and outcome measurement: Blood samples for baseline laboratory tests including hs-CRP were obtained in the emergency room before PCI. Levels of hs-CRP were analyzed by immunoturbidimetric analysis (Tina-quant hs-CRP latex assay, Roche/Hitachi, Cobas, Mannheim, Germany). Twelve-hour fasting serum levels of total cholesterol, triglycerides, and low-density lipoprotein (LDL) and high-density lipoprotein (HDL) cholesterol were measured by standard enzymatic methods. Echocardiograms were performed before or after PCI within 24 hours. Conventional echocardiographic studies including Doppler tissue imaging was performed according to the recommendations of the American Society of Echocardiography. Left ventricular end-systolic and end-diastolic dimensions, interventricular septal and posterior wall thicknesses, and left atrial anteroposterior diameter were determined from 2-dimensional imaging. Ejection fraction was calculated using the biplane Simpson method. We tried to obtain high-frame rate images, and gain settings were adjusted to avoid background noise and to obtain clear Doppler tissue image tracings.

Angiographic procedure and postintervention management: Coronary angiography was performed through either the radial or the femoral artery by a standard technique. Interventional strategies such as the preprocedural utilization of glycoprotein $\mathrm{IIb} / \mathrm{III}$ inhibitors and the use of thrombectomy devices and the performance of postdilatation, intravascular ultrasound, or multivessel stenting were left to the discretion of the individual operator. Bare metal stents (BMS) and drug-eluting stents (DES) were used at the discretion of the individual operator. Successful PCI was defined as TIMI grade 3 flow with residual stenosis $\leq 50 \%$ in the infarct-related artery. During the in-hospital period, patients received essential medical treatment that included $\beta$-blockers, angiotensin-converting enzyme inhibitors, or angiotensin II receptor blockers. After discharge, the patients continued to receive the same optimal medications that they had received in the hospital. Patients were required to visit the outpatient clinic of the cardiology department at the end of the first month, every 6 months after the PCI procedure, and when angina-like symptoms occurred. Therefore, in the present study, we collected clinical follow-up data at one month, 6 months, and 12 months. In our study, the primary end point was composite major adverse cardiac events (MACEs) during a 12-month period. MACEs were defined as the composite of cardiac death, recurrent MI, and target lesion revascularization or coronary artery bypass grafting. Recurrent MI was defined as recurrent symptoms with new ST-segment elevation or reelevation of cardiac markers at least twice the upper limit of normal. Target lesion revascularization was defined as ischemia-induced PCI of the target lesion resulting from restenosis or re-occlusion within the stent or at the adjacent $5 \mathrm{~mm}$ of the distal or proximal segment.

Statistical analysis: All analyses were performed with SPSS for Windows, version 18.0 (SPSS, Inc., Chicago, IL, USA). 
Table I. Baseline Clinical Characteristics of the Patients According to the Level of Hs-CRP and Statin Therapy

\begin{tabular}{|c|c|c|c|c|c|c|}
\hline \multirow{2}{*}{ Variable } & \multicolumn{3}{|c|}{$\begin{array}{c}\text { Low hs-CRP group } \\
(\mathrm{hs}-\mathrm{CRP} \leq 2.0 \mathrm{mg} / \mathrm{L}, n=2,028)\end{array}$} & \multicolumn{3}{|c|}{$\begin{array}{c}\text { High hs-CRP group } \\
(\text { hs-CRP }>2.0 \mathrm{mg} / \mathrm{L}, n=3,091)\end{array}$} \\
\hline & $\begin{array}{l}\text { Without statin } \\
\quad(n=440)\end{array}$ & $\begin{array}{l}\text { With statin } \\
(n=1,588)\end{array}$ & $P$ & $\begin{array}{l}\text { Without statin } \\
\qquad(n=847)\end{array}$ & $\begin{array}{l}\text { With statin } \\
(n=2,244)\end{array}$ & $P$ \\
\hline Age, years & $63.7 \pm 12.9$ & $63.9 \pm 12.5$ & 0.841 & $65.2 \pm 13.0$ & $65.7 \pm 12.3$ & 0.572 \\
\hline Male & $321(73.3)$ & $1,183(74.6)$ & 0.581 & $611(72.2)$ & $1,565(69.8)$ & 0.189 \\
\hline Body mass index, $\mathrm{kg} / \mathrm{m}^{2}$ & $23.8 \pm 3.2$ & $23.9 \pm 3.3$ & 0.572 & $23.5 \pm 3.3$ & $23.9 \pm 3.2$ & 0.061 \\
\hline Killip class III-IV & $75(18.1)$ & $203(13.5)$ & 0.027 & $197(24.0)$ & $389(17.9)$ & $<0.001$ \\
\hline \multicolumn{7}{|l|}{ Medical history } \\
\hline Hypertension & $211(48.7)$ & $712(45.1)$ & 0.178 & $411(48.9)$ & $1,124(50.4)$ & 0.449 \\
\hline Diabetes mellitus & $111(25.7)$ & 409 (25.9) & 0.919 & $262(31.1)$ & 687 (30.9) & 0.913 \\
\hline Smoking & $248(57.7)$ & 977 (62.4) & 0.073 & $476(56.6)$ & $1,304(58.7)$ & 0.302 \\
\hline Hyperlipidemia & $44(10.2)$ & $229(14.5)$ & 0.021 & $51(6.1)$ & $224(10.1)$ & 0.001 \\
\hline Diagnosis & & & 0.124 & & & 0.703 \\
\hline STEMI & $319(72.8)$ & $1,094(69.0)$ & & $588(69.4)$ & $1,553(69.2)$ & \\
\hline NSTEMI & $121(27.2)$ & $494(31.0)$ & & $259(30.6)$ & $691(30.8)$ & \\
\hline \multicolumn{7}{|l|}{ Echocardiographic finding } \\
\hline Left ventricular ejection fraction, $\%$ & $41.9 \pm 7.6$ & $42.3 \pm 7.1$ & 0.306 & $41.5 \pm 8.1$ & $41.5 \pm 7.5$ & 0.781 \\
\hline \multicolumn{7}{|l|}{ Laboratory findings } \\
\hline Maximum troponin $\mathrm{I}, \mathrm{ng} / \mathrm{mL}$ & $5.3 \pm 9.6$ & $6.1 \pm 12.2$ & 0.228 & $6.3 \pm 10.1$ & $6.4 \pm 11.8$ & 0.807 \\
\hline Total cholesterol, mg/dL & $178.9 \pm 45.3$ & $189.1 \pm 43.7$ & $<0.001$ & $173.6 \pm 42.7$ & $183.3 \pm 44.9$ & $<0.001$ \\
\hline Triglyceride, $\mathrm{mg} / \mathrm{dL}$ & $124.9 \pm 91.6$ & $130.0 \pm 102.4$ & 0.325 & $110.1 \pm 67.2$ & $122.6 \pm 96.6$ & $<0.001$ \\
\hline Low-density lipoprotein cholesterol, mg/dL & $112.9 \pm 38.9$ & $121.0 \pm 39.7$ & $<0.001$ & $109.5 \pm 45.7$ & $119.2 \pm 40.1$ & $<0.001$ \\
\hline High-density lipoprotein cholesterol, mg/dL & $46.2 \pm 19.8$ & $45.4 \pm 22.8$ & 0.499 & $43.9 \pm 18.4$ & $43.4 \pm 14.1$ & 0.528 \\
\hline $\mathrm{Hs}-\mathrm{CRP}, \mathrm{mg} / \mathrm{L}$ & $0.7 \pm 0.5$ & $0.7 \pm 0.5$ & 0.534 & $4.2 \pm 2.3$ & $4.0 \pm 2.2$ & 0.250 \\
\hline \multicolumn{7}{|l|}{ Medical therapy during hospitalization } \\
\hline$\beta$-Blocker & $360(81.8)$ & $1,286(81.0)$ & 0.711 & $669(79.0)$ & $1,838(82.0)$ & 0.069 \\
\hline Angiotensin-converting enzyme inhibitor & $299(68.0)$ & $1,117(70.3)$ & 0.214 & $627(74.0)$ & $1,672(74.6)$ & 0.214 \\
\hline Angiotensin receptor blocker & $73(16.7)$ & $294(18.5)$ & 0.385 & $148(17.5)$ & $459(20.5)$ & 0.063 \\
\hline
\end{tabular}

Values are presented as the $n(\%)$ of patients or mean \pm SD. Hs-CRP indicates high-sensitivity C-reactive protein; NSTEMI, Non-ST-segment elevation myocardial infarction; and STEMI, ST-segment elevation myocardial infarction.

Continuous variables are presented as the mean \pm standard deviation and were compared using the unpaired Student $t$ test. Discrete variables are expressed as percentages and frequencies and were compared by chi-square statistics. All statistical tests were 2-tailed with statistical significance defined as a $P \leq$ 0.05 . Univariate analyses using Pearson's chi-square test or Student's $t$ test were performed to evaluate the prognostic significance of demographic and clinical variables for the incidence of MACEs. In addition, the adjusted cumulative incidences of MACEs at 12 months among the groups were analyzed by multivariable Cox regression analysis. The results are presented as adjusted hazard ratios (HRs) with 95\% confidence intervals (CIs) and $P$ values. We further evaluated the effects of the statin therapies on the incidence of MACEs in the different subgroups of patients by Cox regression analysis. Multivariable logistic regression analysis was used to determine independent predictors of 12-month MACEs.

\section{ResUlts}

Baseline clinical characteristics and coronary angiographic and procedural findings: As shown in Table I, the baseline clinical characteristics of the patients were similar among the groups. However, more patients in the low and high hs-CRP without statin groups than with statin groups had a higher Killip class (III to IV). By contrast the low and high hs-CRP with statin groups had more hyperlipidemia patients, and the levels of total cholesterol and LDL cholesterol were higher in the same group. The use of in-hospital medication other than statins was similar among the groups. The most common infarct-related artery was the left anterior descending artery in all groups. There were no significant differences in lesion type according to ACC/AHA classification or initial TIMI flow among the groups. Other coronary angiographic and procedural findings are presented in Table II.

Clinical outcomes at 12 months: The incidence of MACEs was significantly reduced by statin treatment in the total study population, regardless of the level of hs-CRP (without statin group: $17.8 \%$ versus with statin group: $10.8 \%, P<0.001$ ). However, in the low hs-CRP group, the incidence of MACEs did not differ significantly according to statin treatment (without statin group: $12.0 \%$ versus with statin group: $10.1 \%, P=$ 0.249). Meanwhile, the incidence of MACEs was significantly lower in the group treated with statins (without statin group: $20.8 \%$ versus with statin group: $11.3 \%, P<0.001$ ), which was mainly due to the decreased cardiac death rate $(9.8 \%$ versus $6.6 \%, P=0.003$ ) (Table III).

The adjusted cumulative incidences of MACEs during the 12 months among the groups according to multivariable Cox regression analysis are presented in Figure 2. The incidences of MACEs at 12 months were not significantly different between the low hs-CRP with statin group and the low hsCRP without statin group (Figure 2A). However, the incidences of MACEs were reduced by statin treatment in the high hs-CRP group (Figure 2B). This trend was also found in patients with an LVEF less than 40\% (Figure 3A, B).

In another subgroup analysis, in the high hs-CRP group, statin treatment significantly reduced the incidence of MACEs in all subgroups, while in the low hs-CRP group, statin treat- 
Table II. Baseline Coronary Angiographic and Procedural Characteristics of the Patients According to the Level of Hs-CRP and Statin Therapy

\begin{tabular}{|c|c|c|c|c|c|c|}
\hline \multirow{2}{*}{ Parameter } & \multicolumn{3}{|c|}{$\begin{array}{c}\text { Low hs-CRP group } \\
(\text { hs-CRP } \leq 2.0 \mathrm{mg} / \mathrm{L}, n=2,028)\end{array}$} & \multicolumn{3}{|c|}{$\begin{array}{c}\text { High hs-CRP group } \\
(\text { hs-CRP }>2.0 \mathrm{mg} / \mathrm{L}, n=3,091)\end{array}$} \\
\hline & $\begin{array}{l}\text { Without statin } \\
\qquad(n=440)\end{array}$ & $\begin{array}{l}\text { With statin } \\
(n=1,588)\end{array}$ & $P$ & $\begin{array}{l}\text { Without statin } \\
\qquad(n=847)\end{array}$ & $\begin{array}{l}\text { With statin } \\
(n=2,244)\end{array}$ & $P$ \\
\hline Infarct-related coronary artery & & & 0.302 & & & 0.536 \\
\hline Left anterior descending artery & $254(57.7)$ & $924(58.2)$ & & $491(58.0)$ & $1,310(47.4)$ & \\
\hline Right coronary artery & $124(28.2)$ & $398(25.1)$ & & $232(27.4)$ & $568(25.3)$ & \\
\hline Left circumflex artery & $57(13.0)$ & $234(14.7)$ & & $106(12.5)$ & $316(14.1)$ & \\
\hline Left main & $5(1.1)$ & $32(2.0)$ & & $18(2.1)$ & $50(2.2)$ & \\
\hline Multivessel coronary disease & $232(52.7)$ & $856(53.9)$ & 0.661 & $506(59.7)$ & $1,312(58.5)$ & 0.521 \\
\hline Lesion type $\mathrm{B}_{2}-\mathrm{C}$ & $291(66.1)$ & $1,062(66.9)$ & 0.701 & $625(73.8)$ & $1,702(75.8)$ & 0.209 \\
\hline Initial TIMI flow grade 0 & $208(51.0)$ & $774(52.7)$ & 0.138 & $393(26.3)$ & $1,104(51.2)$ & 0.230 \\
\hline \multicolumn{7}{|l|}{ Stent } \\
\hline Drug-eluting stent & $387(87.9)$ & $1,353(85.2)$ & 0.334 & $680(80.3)$ & $1,877(83.6)$ & 0.119 \\
\hline Stent size, $\mathrm{mm}$ & $23.4 \pm 7.1$ & $23.5 \pm 7.9$ & 0.773 & $24.8 \pm 6.6$ & $25.0 \pm 6.6$ & 0.370 \\
\hline Stent diameter, $\mathrm{mm}$ & $3.2 \pm 0.4$ & $3.2 \pm 0.4$ & 0.793 & $3.2 \pm 0.4$ & $3.1 \pm 0.4$ & 0.123 \\
\hline Number of stents & $1.4 \pm 0.8$ & $1.5 \pm 0.8$ & 0.063 & $1.6 \pm 0.8$ & $1.6 \pm 0.9$ & 0.293 \\
\hline GP IIb/IIIa inhibitor use during PCI & $58(13.2)$ & $251(15.8)$ & 0.175 & $111(13.1)$ & $308(13.7)$ & 0.653 \\
\hline
\end{tabular}

GP indicates glycoprotein; hs-CRP, high sensitivity C-reactive protein; PCI, percutaneous coronary intervention; and TIMI, Thrombolysis in Myocardial Infarction.

Table III. Clinical Outcomes at 12-months

\begin{tabular}{|c|c|c|c|c|c|c|}
\hline \multirow{2}{*}{ Outcomes } & \multicolumn{3}{|c|}{$\begin{array}{c}\text { Low hs-CRP group } \\
\text { (hs-CRP } \leq 2.0 \mathrm{mg} / \mathrm{L}, n=2,028 \text { ) }\end{array}$} & \multicolumn{3}{|c|}{$\begin{array}{c}\text { High hs-CRP group } \\
\text { (hs-CRP }>2.0 \mathrm{mg} / \mathrm{L}, n=3,091)\end{array}$} \\
\hline & $\begin{array}{l}\text { Without statin } \\
\qquad(n=440)\end{array}$ & $\begin{array}{l}\text { With statin } \\
(n=1,588)\end{array}$ & $P$ & $\begin{array}{l}\text { Without statin } \\
\qquad(n=847)\end{array}$ & $\begin{array}{l}\text { With statin } \\
(n=2,244)\end{array}$ & $P$ \\
\hline Composite & $53(12.0)$ & $161(10.1)$ & 0.249 & $176(20.8)$ & $253(11.3)$ & $<0.001$ \\
\hline Cardiac death & $21(4.8)$ & $68(4.3)$ & 0.657 & $83(9.8)$ & $148(6.6)$ & 0.003 \\
\hline Re-myocardial infarction & $6(1.4)$ & $21(1.3)$ & 0.947 & $17(2.0)$ & $30(1.3)$ & 0.174 \\
\hline Target lesion revascularization & $34(7.7)$ & $97(6.1)$ & 0.222 & $72(8.5)$ & $152(6.8)$ & 0.099 \\
\hline Coronary artery bypass graft & $2(0.5)$ & $6(0.4)$ & 0.820 & $5(0.6)$ & $3(0.1)$ & 0.026 \\
\hline
\end{tabular}

Hs-CRP indicates high-sensitivity C-reactive protein.

A

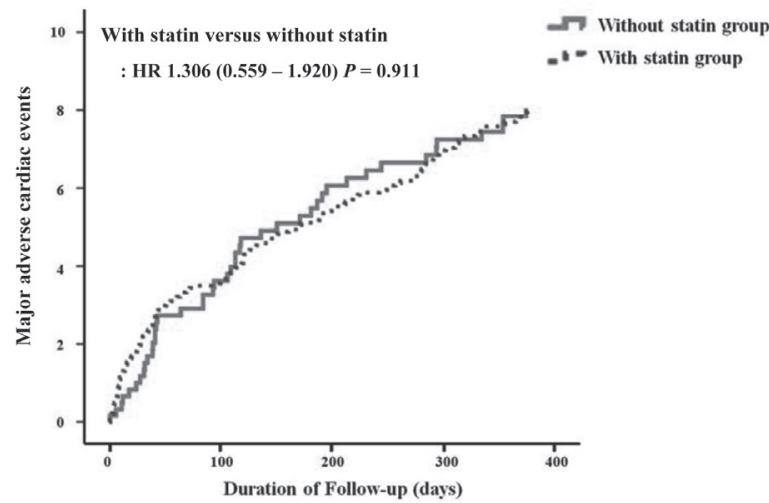

B

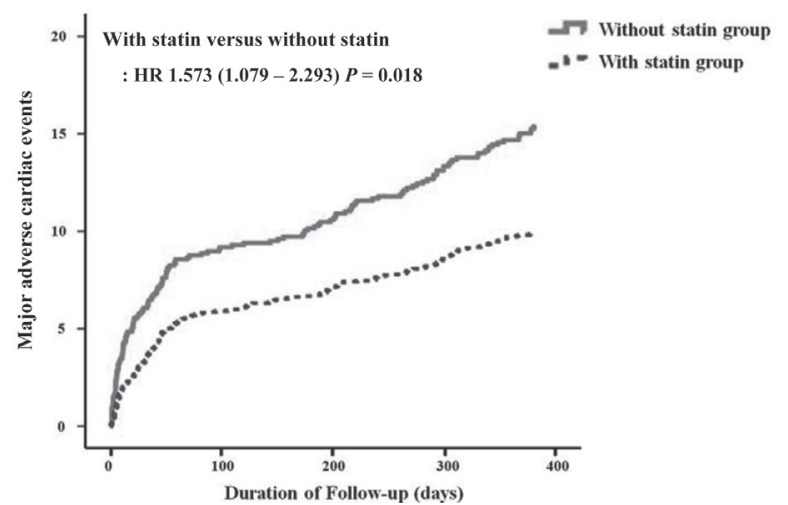

Figure 2. Adjusted cumulative incidences of major adverse cardiac events (MACEs) at 12 months in the low hs-CRP group (A) and the high hs-CRP group (B). Variables in the multivariable Cox regression analysis. hs-CRP indicates high-sensitivity C-reactive protein and HR, hazard ratio.

ment did not significantly influence the incidence of MACEs in any subgroup (Figure 4).

Multivariate logistic regression analysis was performed to identify the predictors of MACEs. In the low hs-CRP group, Killip class III to IV, hypertension, and age $\geq 65$ years were independent predictors of MACEs after adjustment for the clinical characteristics in model 1. In model 2, Killip class III to IV, hypertension, and multivessel disease were independent pre- dictors of MACEs after adjustment for angiographic and procedural data in addition to clinical characteristics. In the high hs-CRP group, lack of statin treatment, Killip class III to IV, age $\geq 65$ years, and lack of beta-blocker treatment were independent predictors of MACEs in model 1, whereas lack of statin treatment, Killip class III to IV, and age $\geq 65$ years were independent predictors of MACEs in model 2 (Table IV). 
A

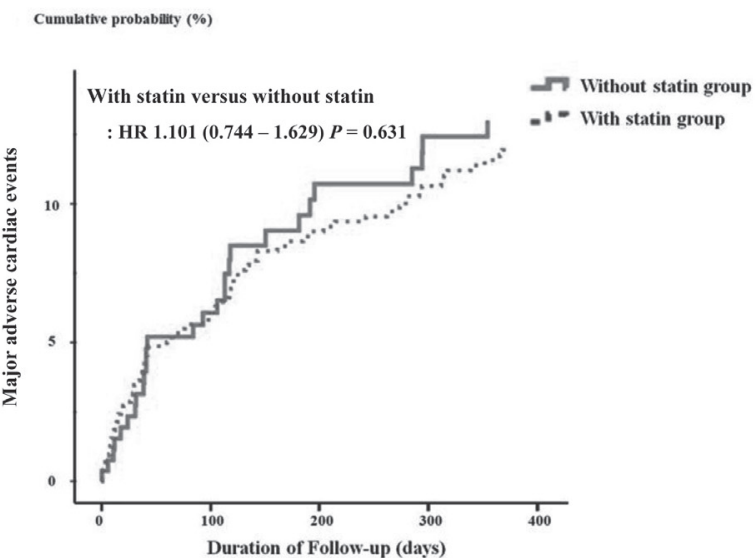

B

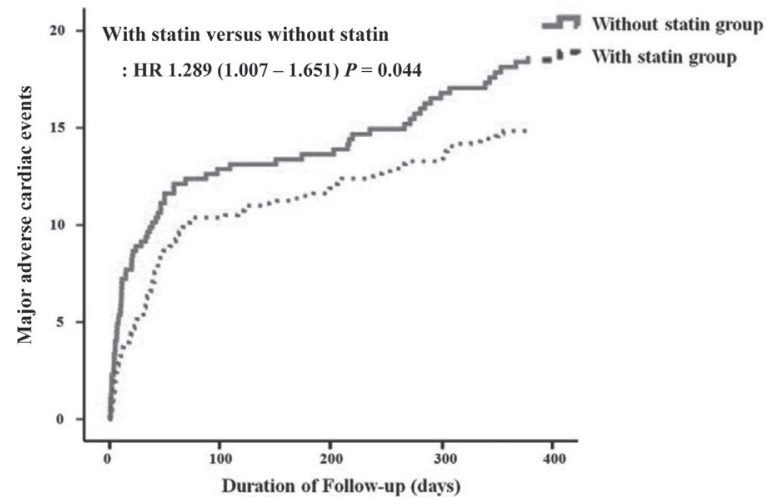

Figure 3. Adjusted cumulative incidences of major adverse cardiac events (MACEs) at 12 months in the subgroup of patients with left ventricular ejection fraction less than $40 \%$. (A) Low high-sensitivity C-reative protein (hs-CRP) group; (B) high hs-CRP group.

\section{Low hs-CRP group}

In all patients
In age $<65$ patients
In age $\geq 65$ patients
In diabetic patients
In non-diabetic patients
In Killip class I-II patients
In Killip class III-IV patients
In LDL $<100 \mathrm{mg} / \mathrm{dL}$ patients
In LDL $\geq 100 \mathrm{mg} / \mathrm{dL}$ patients
In STEMI patients
In NSTEMI patients

\section{DISCUSSION}

In the current study, we investigated the effects of statins on long-term clinical outcomes in AMI patients with decreased LV systolic function. The major finding of our study was that statin therapy significantly reduced the incidence of MACEs, including cardiac death, in AMI patients with elevated levels of hs-CRP, but not in AMI patients with low levels of hs-CRP.

Clinical implications of the study: The pleotropic effects of statins, independent of their lipid-lowering effect, have been described to reduce plaque thrombogenicity, improve endothelial function, and inhibit free-radical production in the vascular wall. ${ }^{16)}$ Moreover, statins have potent anti-inflammatory effects on myocardium as well as beneficial effects in AMI and LV

\section{High hs-CRP group}

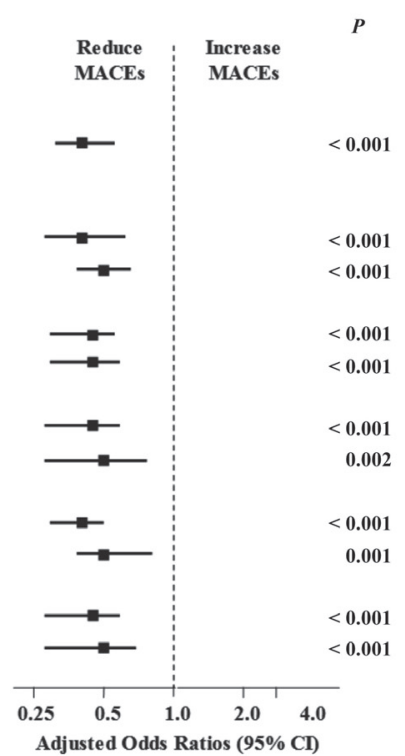

Figure 4. Subgroup analysis. Adjusted odds ratios (ORs) for the incidence of major adverse cardiac events (MACEs) associated with statin therapy in the entire study population and in various subgroups of patients according to age, diabetes mellitus, Killip class, level of low-density lipoprotein (LDL) cholesterol, and final diagnosis. STEMI indicates ST-segment elevation myocardial infarction and NSTEMI, non-ST-segment elevation myocardial infarction.

dysfunction patients, such as improvements in symptom severity, B natriuretic peptide (BNP) levels, and endothelial function. ${ }^{17,18)}$ Nevertheless, the effect of statins on the clinical prognosis of LV dysfunction patients remains equivocal. The first important randomized clinical trial on statins in LV dysfunction patients was The Controlled Rosuvastatin Multinational Trial in Heart Failure (CORONA) study. ${ }^{19)}$ A total of 5,091 patients with ischemic systolic heart failure (NYHA class II-IV) were randomized to receive rosuvastatin $(10 \mathrm{mg} / \mathrm{day})$ or placebo and were followed-up prospectively for a median of 2.7 years. Despite significantly decreasing LDL cholesterol and CRP levels and reducing cardiovascular hospitalizations, rosuvastatin failed to reduce the primary outcome (death from cardiovascular causes, nonfatal myocardial infarction, or nonfatal 
Table IV. Multivariate Logistic Regression Analysis for Predicting Major Adverse Cardiac Events

\begin{tabular}{|c|c|c|c|c|c|c|}
\hline \multirow{2}{*}{ Variable } & \multicolumn{2}{|c|}{ Unadjusted Model } & \multicolumn{2}{|l|}{ Model $1^{*}$} & \multicolumn{2}{|l|}{ Model 2** } \\
\hline & $\mathrm{HR}(95 \% \mathrm{CI})$ & $P$ & $\mathrm{HR}(95 \% \mathrm{CI})$ & $P$ & $\mathrm{HR}(95 \% \mathrm{CI})$ & $P$ \\
\hline \multicolumn{7}{|l|}{ Low hs-CRP group } \\
\hline Killip class III-IV & $6.253(3.718-10.515)$ & $<0.001$ & $2.797(1.704-4.591)$ & $<0.001$ & $3.591(2.075-6.213)$ & $<0.001$ \\
\hline Hypertension & $2.140(1.259-3.636)$ & 0.004 & $2.100(1.305-3.379)$ & 0.002 & $2.508(1.460-4.307)$ & 0.001 \\
\hline Multivessel disease & $1.801(1.048-3.097)$ & 0.031 & & & $1.797(1.044-3.095)$ & 0.034 \\
\hline Age $\geq 65$ years & $3.865(2.081-7.179)$ & $<0.001$ & $1.503(1.075-2.102)$ & 0.017 & $1.336(0.775-2.410)$ & 0.281 \\
\hline Lack of beta-blocker treatment & $2.915(1.733-4.902)$ & $<0.001$ & $1.041(0.601-1.804)$ & 0.961 & $1.099(0.591-2.044)$ & 0.765 \\
\hline Lack of statin treatment & $1.214(0.873-1.689)$ & 0.294 & $1.089(0.635-1.869)$ & 0.756 & $1.306(0.559-1.920)$ & 0.911 \\
\hline \multicolumn{7}{|l|}{ High hs-CRP group } \\
\hline Lack of statin treatment & $1.733(1.263-2.375)$ & $<0.001$ & $1.651(1.168-2.333)$ & 0.004 & $1.573(1.079-2.293)$ & 0.018 \\
\hline Killip class III-IV & $2.922(2.121-4.025)$ & $<0.001$ & $1.722(1.188-2.497)$ & 0.004 & $1.615(1.077-2.421)$ & 0.020 \\
\hline Age $\geq 65$ years & $4.144(2.731-6.288)$ & $<0.001$ & $1.567(1.047-2.346)$ & 0.029 & $1.688(1.087-2.623)$ & 0.020 \\
\hline Lack of beta-blocker treatment & $2.136(1.546-2.949)$ & $<0.001$ & $1.521(1.014-2.282)$ & 0.043 & $1.478(0.943-2.315)$ & 0.088 \\
\hline STEMI & $1.336(0.975-1.832)$ & 0.071 & $1.432(1.006-2.040)$ & 0.046 & $1.393(0.928-2.090)$ & 0.109 \\
\hline Multivessel disease & $1.775(1.267-2.486)$ & 0.001 & & & $1.149(0.782-1.690)$ & 0.479 \\
\hline
\end{tabular}

* Model 1 was adjusted for clinical characteristics ** Model 2 was adjusted for clinical characteristics, angiographic findings, and procedural data. CI indicates confidence interval; hs-CRP, high-sensitivity C-reactive protein; and HR, hazard ratio.

stroke) or the number of deaths from any other cause. The GISSI-HF (Gruppo Italiano per lo Studio della Sopravvivenza nell'Insufficienza Cardiaca) study was another placebo-controlled randomized clinical trial in 4,574 heart failure patients that yielded similar results. ${ }^{20)}$ In this trial, which also included patients with nonischemic and/or diastolic heart failure (NYHA class II-IV), rosuvastatin (10 mg/day) failed to improve cardiovascular outcomes after a median of 3.9 years. Notably, statin administration could also have adverse effects in LV dysfunction patients given the association between low cholesterol levels and poor outcome in advanced LV dysfunction. Other considerations contraindicating statin use in LV dysfunction include their adverse effects on coenzyme Q10 (ubiquinone) and selenoproteins that are important for myocardium biology. ${ }^{21-23)}$ However, McMurray, et al retrospectively analyzed the CORONA study to examine outcomes in patients with heart failure in relation to hs-CRP concentrations and the effect of statin therapy. ${ }^{12)}$ They compared $10 \mathrm{mg}$ rosuvastatin daily with placebo in 4,961 patients with ischemic systolic LV dysfunction according to a baseline hs-CRP $<2.0 \mathrm{mg} / \mathrm{L}$ or $\geq$ $2.0 \mathrm{mg} / \mathrm{L}$. The result was that more placebo-treated patients had a primary end point in the high hs-CRP group and in the low hs-CRP group, however, the incidence of primary end point was not significantly different between the placebo and rosuvastatin groups. To the best of our knowledge, this is the only study that has described an interaction between hs-CRP and the effect of statins. The effect of statins in AMI patients with left ventricular dysfunction according to the level of hsCRP has not been reported. ${ }^{24)}$

To our knowledge, the present study is the first to show that statin therapy can improve the long-term clinical outcomes in AMI patients with LV systolic dysfunction who were prone to high inflammatory status. The findings suggest that statin therapy was beneficial in the group of patients with baseline hs-CRP levels $\geq 2.0 \mathrm{mg} / \mathrm{L}$. No such benefit was observed in the group of patients with hs-CRP $<2.0 \mathrm{mg} / \mathrm{L}$. One cause of this controversy is presumed to be that the clinical trials described above did not consider the concept of inflammation. It is well known that local and systemic inflammation plays a pivotal role in the pathogenesis of AMI and LV dysfunction. Hs-CRP, an inflammatory biomarker that independently predicts future vascular events, improves global classification of risk. ${ }^{25)}$ These findings indicate that there is a group of heart failure patients that may indeed benefit from statin treatment. An explanation for these findings is that statins might exert beneficial effects only in the early stages of the CVD continuum, as in low risk subjects without CVD and early stage heart failure patients, and in high risk patients with end-stage heart failure. Thus, we believe that early prescription of statin therapy in the present study might have played an additional role in the protection of AMI patients with decreased LV systolic function who have high levels of hs-CRP, and thereby decreased the prevalence of MACEs. Also, hs-CRP level is particularly helpful in detecting those heart failure patients more likely to benefit from statin treatment.

Some aspects of the methodology of our study should be compared with previous studies. First, we applied a $2 \mathrm{mg} / \mathrm{L}$ cutoff value of hs-CRP. A potential clinical hs-CRP cutoff value of $2 \mathrm{mg} / \mathrm{L}$ is supported by studies in the Justification for the Use of Statins in Prevention: an Intervention Trial Evaluating Rosuvastatin (JUPITER) and Atherosclerosis Risk in Communities (ARIC) populations. ${ }^{26-29)}$ Second, according to the lowgrade inflammation concept, we excluded 430 patients with levels $\geq 10 \mathrm{mg} / \mathrm{L}$ because infections, trauma, and acute illness have been associated with increases in hs-CRP levels. ${ }^{30)} \mathrm{We}$ therefore believe that measured hs-CRP levels largely correspond to a patient's baseline grade of inflammation, but we cannot exclude the possibility that some patients experienced slight increases in hs-CRP levels $<10 \mathrm{mg} / \mathrm{L}$ because of acute disease.

Study limitations: First, although our study included a large number of subjects, because of the nonrandomized nature of the registry study, there were baseline differences in several important prognostic factors between our primary comparison groups. Although we included most confounders in the multivariable Cox regression model to control for the baseline biases, it is possible that some potential confounders may have crept in. For example, those taking statins are less severely compromised (lower proportion of Killip III-IV, less anterior descending coronary artery as culprit vessel) and might have a better outcome. However, this multicenter registry may help complete the picture gained from randomized trials, which 
usually have highly selected patients treated in a nonroutine setting. Therefore, our study is of value because it used registry data, which reflects the real world. Second, our study did not present the exact criteria for statin therapy, and did not provide a clear rationale for the patients not treated with statins. Total cholesterol and LDL levels were significantly higher in the statin treatment group, irrespective of hs-CRP levels. Statins might have been prescribed based on the medical insurance of our country. Third, the type and dose of statins were not controlled in our study and the changes were not evaluated. Fourth, other medications which can affect hs-CRP levels, such as non-steroidal anti-inflammatory drugs, were not evaluated. Finally, the follow-up hs-CRP and cholesterol levels were not evaluated. Therefore, a prospective randomized controlled trial is required to confirm this apparent therapeutic effect of statins in AMI patients with LV systolic dysfunction.

Conclusions: In conclusion, the present study showed that statin treatment had an effect on long-term clinical outcomes in AMI patients with decreased LV systolic function who had high levels of hs-CRP. Therefore, these results indicate that the hs-CRP level is particularly helpful in detecting AMI patients with LV dysfunction who are more likely to benefit from statin treatment.

\section{REFERENCES}

1. Anzai T. Post-infarction inflammation and left ventricular remodeling- a double-edged sword. Circ J 2013; 77: 580-7. (Review)

2. Lee WW, Marinelli B, van der Laan AM, et al. PET/MRI of inflammation in myocardial infarction. J Am Coll Cardiol 2012; 59: 153-63.

3. Voulgari C, Tentolouris N, Dilaveris P, Tousoulis D, Katsilambros $\mathrm{N}$, Stefanadis C. Increased heart failure risk in normal-weight people with metabolic syndrome compared with metabolically healthy obese individuals. J Am Coll Cardiol 2011; 58: 1343-50.

4. van Diepen S, Roe MT, Lopes RD, et al. Baseline NT-proBNP and biomarkers of inflammation and necrosis in patients with STsegment elevation myocardial infarction: insights from the APEXAMI trial. J Thromb Thrombolysis 2012; 34: 106-13.

5. Mano Y, Anzai T, Kaneko H, et al. Overexpression of human Creactive protein exacerbates left ventricular remodeling in diabetic cardiomyopathy. Circ J 2011; 75: 1717-27.

6. Gabay C, Kushner I. Acute-phase proteins and other systemic responses to inflammation. N Engl J Med 1999; 340: 448-54. (Review)

7. Rossi IA, Bochud M, Viswanathan B, Riesen W, Bovet P. Relation between high-sensitivity C-reactive protein and cardiovascular and renal markers in a middle-income country in the African region. Int J Cardiol 2012; 156: 203-8.

8. Davignon J. Beneficial cardiovascular pleiotropic effects of statins. Circulation 2004; 109: III39-43. (Review)

9. Zhou Q, Liao JK. Pleiotropic effects of statins. - Basic research and clinical perspectives -. Circ J 2010; 74: 818-26. (Review)

10. Horwich TB, Hamilton MA, Maclellan WR, Fonarow GC. Low serum total cholesterol is associated with marked increase in mortality in advanced heart failure. J Card Fail 2002; 8: 216-24.

11. Rauchhaus M, Clark AL, Doehner W, et al. The relationship between cholesterol and survival in patients with chronic heart failure. J Am Coll Cardiol 2003; 42: 1933-40.

12. McMurray JJ, Kjekshus J, Gullestad L, et al. Effects of statin therapy according to plasma high-sensitivity C-reactive protein concentration in the Controlled Rosuvastatin Multinational Trial in Heart Failure (CORONA): a retrospective analysis. Circulation 2009; 120: 2188-96.

13. Krum H, McMurray JJ. Statins and chronic heart failure: do we need a large-scale outcome trial? J Am Coll Cardiol 2002; 39: $1567-73$.

14. Velavan P, Huan Loh P, Clark A, Cleland JG. The cholesterol paradox in heart failure. Congest Heart Fail 2007; 13: 336-41. (Review)

15. Jeong $\mathrm{HC}$, Ahn $\mathrm{Y}$, Jeong $\mathrm{MH}$, et al. Intensive pharmacologic treatment in patients with acute non ST-segment elevation myocardial infarction who did not undergo percutaneous coronary intervention. Circ J 2008; 72: 1403-9.

16. Ito MK, Talbert RL, Tsimikas S. Statin-associated pleiotropy: possible beneficial effects beyond cholesterol reduction. Pharmacotherapy 2006; 26: 85S-97S. (Review)

17. Tousoulis D, Antoniades C, Bosinakou E, et al. Effects of atorvastatin on reactive hyperaemia and the thrombosis-fibrinolysis system in patients with heart failure. Heart 2005; 91: 27-31.

18. Node K, Fujita M, Kitakaze M, Hori M, Liao JK. Short-term statin therapy improves cardiac function and symptoms in patients with idiopathic dilated cardiomyopathy. Circulation 2003; 108: 839-43.

19. Kjekshus J, Apetrei E, Barrios V, et al. Rosuvastatin in older patients with systolic heart failure. N Engl J Med 2007; 357: 224861.

20. Tavazzi L, Maggioni AP, Marchioli R, et al. Effect of rosuvastatin in patients with chronic heart failure (the GISSI-HF trial): a randomised, double-blind, placebo-controlled trial. Lancet 2008; 372: 1231-9.

21. Ashton E, Windebank E, Skiba M, et al. Why did high-dose rosuvastatin not improve cardiac remodeling in chronic heart failure? Mechanistic insights from the UNIVERSE study. Int J Cardiol 2011; 146: 404-7.

22. Young JM, Molyneux SL, Reinheimer AM, et al. Relationship between plasma coenzyme Q10, asymmetric dimethylarginine and arterial stiffness in patients with phenotypic or genotypic familial hypercholesterolemia on long-term statin therapy. Atherosclerosis 2011; 218: 188-93

23. Ramasubbu K, Estep J, White DL, Deswal A, Mann DL. Experimental and clinical basis for the use of statins in patients with ischemic and nonischemic cardiomyopathy. J Am Coll Cardiol 2008; 51: 415-26. (Review)

24. Sakamoto T, Kojima S, Ogawa H, et al. Effects of early statin treatment on symptomatic heart failure and ischemic events after acute myocardial infarction in Japanese. Am J Cardiol 2006; 97: 1165-71.

25. Lim SY, Jeong MH, Bae EH, et al. Predictive factors of major adverse cardiac events in acute myocardial infarction patients complicated by cardiogenic shock undergoing primary percutaneous coronary intervention. Circ J 2005; 69: 154-8.

26. Mora S, Glynn RJ, Boekholdt SM, Nordestgaard BG, Kastelein JJ, Ridker PM. On-treatment non-high-density lipoprotein cholesterol, apolipoprotein B, triglycerides, and lipid ratios in relation to residual vascular risk after treatment with potent statin therapy: JUPITER (justification for the use of statins in prevention: an intervention trial evaluating rosuvastatin). J Am Coll Cardiol 2012; 59: 1521-8.

27. Peña JM, MacFadyen J, Glynn RJ, Ridker PM. High-sensitivity C-reactive protein, statin therapy, and risks of atrial fibrillation: an exploratory analysis of the JUPITER trial. Eur Heart J 2012; 33: 531-7.

28. Ridker PM, Danielson E, Fonseca FA, et al. Rosuvastatin to prevent vascular events in men and women with elevated C-reactive protein. N Engl J Med 2008; 359: 2195-207.

29. Yang EY, Nambi V, Tang Z, et al. Clinical implications of JUPITER (Justification for the Use of statins in Prevention: an Intervention Trial Evaluating Rosuvastatin) in a U.S. population: insights from the ARIC (Atherosclerosis Risk in Communities) study. J Am Coll Cardiol 2009; 54: 2388-95.

30. Ridker PM. Clinical application of C-reactive protein for cardiovascular disease detection and prevention. Circulation 2003; 107: 363-9. (Review) 